\title{
Sur les espaces de Musielak-Orlicz non localement convexes intransitifs
}

par

\author{
ALBERT K. KALINDE (Lusaka)
}

Sommaire. Soient $\Omega$ un espace mesuré muni d'une mesure $\sigma$-finie sans atome et $\varphi$ une fonction de Musielak-Orlicz sur $\Omega$. En convenant de noter par $L_{0}^{\varphi}(\Omega)$ le sous-espace de l'espace de Musielak-Orlicz $L^{\varphi}(\Omega)$ associé à $\varphi$, constitué de fonctions scalaires mesurables $u$ définies sur $\Omega$ à une fonction négligeable près pour lesquelles les $\lambda u$ sont $\varphi$-intégrables quel que soit le réel $\lambda$ strictement positif, nous examinons d'abord à quelle condition tout opérateur linéaire continu de $L_{0}^{\varphi^{0}}\left(\Omega_{0}\right)$ dans $L_{0}^{\varphi_{0}^{1}}\left(\Omega_{1}\right)$ est nul, où $\varphi^{1}(t, x)$ est une fonction de $t$ concave pour tout $x$ pris dans $\Omega_{1}$. Nous appliquons ensuite ce résultat à la caractérisation des sous-espaces fermés non triviaux invariants par rapport à tous les endomorphismes continus d'une classe d'espaces de MusielakOrlicz généralement non localement convexes $L_{0}^{\mathscr{Q}}(\Omega)$, où $\Omega$ est un espace métrique complet séparable dont la mesure est positive, bornée, régulière et sans atome.

\$ 0. Introduction. En considérant un espace mesuré $\Omega$ de mesure sans atome, Ph. Turpin (cf. [14], théorème 3.4.8) a établi que $\varrho_{1}(t)$ et $\varrho_{0}(t)$ étant des fonctions d'Orlicz respectivement concave et vérifiant la condition $\Delta_{2}$ (cf. [13]), s'il existe un opérateur linéaire continu non nul de $L^{\ell_{0}}(\Omega)$ dans $L^{\ell_{1}}(\Omega)$, alors $\lim \sup \varrho_{1}(t) / \varrho_{0}(t)<+\infty$.

Pour une fonction de Musielak-Orlicz $\varphi$ sur $\Omega$, notons $L_{0}^{\varphi}(\Omega)$ le sousespace de l'espace de Musielak-Orlicz $L^{\varphi}(\Omega)$ formé de fonctions scalaires mesurables $u$ sur $\Omega$, définies à une fonction négligeable près, telles que les $\lambda u$ soient $\varphi$-intégrables sur $\Omega$ quel que soit le réel $\lambda$ strictement positif. L'ensemble des fonctions simples contenues dans $L_{0}^{\varphi}(\Omega)$ est dense dans ce sousespace. Dans ce travail, nous nous limitons essentiellement à ces sous-espaces et les appelons espaces de Musielak-Orlicz.

Lorsque $\varphi^{0}$ et $\varphi^{1}$ sont des fonctions de Musielak-Orlicz respectivement sur les espaces mesurés $\Omega_{0}$ et $\Omega_{1}$ de mesures $\sigma$-finies sans atome, où $\varphi^{1}(t, x)$ est une fonction de $t$ concave pour tout $x$ dans $\Omega_{1}$, dans le théorème 2.3 nous généralisons aux espaces de Musielak-Orlicz le résultat de Turpin mentionné ci-dessus, en démontrant que si $\varphi^{0}$ et $\varphi^{1}$ satisfont aux conditions $\mathrm{C}_{0}$ et $\mathrm{C}_{1}$ respectivement (cf. définition 9), alors l'existence d'un couple $(x, y)$ dans $\Omega_{0} \times \Omega_{1}$ tel que $\lim \sup \varphi^{1}(t, y) / \varphi^{0}(t, x)<+\infty$ est une condition nécessaire d'existence d'un opérateur linéaire continu non nul de $L_{0}^{\varphi^{0}}\left(\Omega_{0}\right)$ dans $L_{0}^{\varphi^{1}}\left(\Omega_{1}\right)$. 
Dans le cas où $\varphi^{0}$ et $\varphi^{1}$ sont des fonctions de Musielak-Orlicz quelconques sur respectivement les espaces métriques complets séparables $\Omega_{0}$ et $\Omega_{1}$ dont les mesures sont bornées, sans atome et régulières, la condition $\limsup \varphi^{1}(t, y) / \varphi^{0}(t, x)<+\infty$ pour tout couple $(x, y)$ dans $\Omega_{0} \times \Omega_{1}$ a

comme conséquence dans le corollaire 3.2 que lorsque $\varphi^{0}$ et $\varphi^{1}$, puis $\Omega_{0}$ et $\Omega_{1}$ sont identiques respectivement à $\varphi$ et $\Omega$, l'espace de Musielak-Orlicz $L_{0}(\Omega)$ est transitif, c'est-à-dire qu'il n'admet comme sous-espaces fermés invariants par rapport à tous ses endomorphismes continus que les sousespaces triviaux. Comme cas particulier, tout espace d'Orlicz $L^{\ell}([0,1])$ engendré par une fonction d'Orlicz $\varrho$ vérifiant la condition $\Delta_{2}$ (cf. définition 2) est aussi transitif.

Quand $\varphi$ est une fonction de Musielak-Orlicz sur un espace métrique complet séparable muni d'une mesure positive $\sigma$-additive bornée, régulière et sans atome, de plus telle que $\varphi(t, x)$ est une fonction de $t$ concave pour tout $x$ dans $\Omega$ et vérifie les conditions $\mathrm{C}_{0}$ et $\mathrm{C}_{1}$, nous montrons dans le théorème 3.5 que les seuls sous-espaces fermés non triviaux de $L_{0}^{\varphi}(\Omega)$ invariants par rapport à tous les endomorphismes continus de cet espace sont du type $L_{0}^{\varphi}(H)$, où $H$ est un ensemble mesurable de mesure strictement positive plus petite strictement que celle de $\Omega$ et tel que pour tout $y$ dans $H$, l'ensemble des $x$ dans $\Omega$ vérifiant la condition $\limsup \varphi(t, x) / \varphi(t, y)<+\infty$ est entièrement contenu dans $H$.

$$
\rightarrow \rightarrow \infty
$$

Cette note fait suite à l'article [17] dans lequel fut construit pour la première fois un espace vectoriel topologique rigide, c'est-à-dire dont les seuls endomorphismes continus sont des multiples de lidentité par les nombres complexes.

\section{$\S 1$. Preliminaires.}

DÉfinition 1. On appelle fonction d'Orlicz (cf. [15]) toute application $\varrho$ de $[0,+\infty]$ dans $[0,+\infty]$ non identiquement nulle, croissante, continue à gauche sur $(0,+\infty]$, nulle et continue en 0 .

Définition 2. Une fonction d'Orlicz $\varrho$ vérifie la condition $\Delta_{\mathbf{2}}$ (cf. [13]) partout (respectivement au voisinage de 0 , de $+\infty$ ) lorsqu'il existe une constante $m$ strictement positive telle que $\varrho(2 t) \leqslant m \varrho(t)$ pour tout $t$ (respectivement pour $t$ dans le voisinage de 0 , de $+\infty$ ).

DÉFINITION 3. Étant donné un espace mesuré $\Omega$, c'est-à-dire un ensemble non vide muni d'une tribu de parties ainsi que d'une mesure positive $\sigma$ additive, une fonction de Musielak-Orlicz $\varphi$ sur $\Omega$ (cf. [9]) est une application de $[0,+\infty] \times \Omega$ dans $[0,+\infty]$ telle que pour chaque $x$ fixé dans $\Omega, \varphi(t, x)$ est une fonction d'Orlicz et pour chaque $t \geqslant 0$ fixé, $\varphi(t, x)$ est une fonction de $x$ mesurable sur $\Omega$.

Remarque 1 . Dans cet article, les mesures considérées sont toutes positives non nulles et $\sigma$-additives.
Défintrion 4. Soient $\Omega$ un espace mesuré de mesure $\mu, \varphi$ une fonction de Musielak-Orlicz sur $\Omega$ et $\mathscr{M}_{\Omega}$ l'ensemble des (classes de) fonctions scalaires mesurables, définies à une fonction négligeable près et finies presque partout.' L'ensemble

$$
L^{\varphi}(\Omega)=\left\{u \in \mathscr{M}_{\Omega}: \exists \varepsilon>0, \int_{\Omega} \varphi(\varepsilon|u(x)|, x) d \mu<+\infty\right\}
$$

est un espace vectoriel. Lorsqu'il est muni de la topologie vectorielle métrisable engendrée par la $F$-norme (cf. [15])

$$
v_{\varphi}(u)=\inf \left\{\varepsilon>0: \int_{\Omega} \varphi(|u(x)| / \varepsilon, x) d \mu \leqslant \varepsilon\right\},
$$

il est appelé espace de Musielak-Orlicz. Il est complet pour cette topologie dont une base de voisinages de 0 est constituée des ensembles $\varepsilon B_{\Omega}^{\varphi}(\varepsilon)$ avec $\varepsilon>0$, où

$$
B_{\Omega}^{\varphi}(\varepsilon)=\left\{u \in L^{\varphi}(\Omega): \int_{\Omega} \varphi(|u(x)|, x) d \mu \leqslant \varepsilon\right\} .
$$

Lorsque $\varphi(t, x)$ est une fonction de $t$ concave pour tout $x$ dans $\Omega, L^{\varphi}(\Omega)$ n'est généralement pas localement convexe.

Remarque 2. Il est important de noter que pour toute fonction de Musielak-Orlicz $\varphi$ sur $\Omega$, par espace de Musielak-Orlicz associé à $\varphi$ nous entendons dans cette note essentiellement le sous-espace de $L^{\varphi}(\Omega)$ formé de fonctions $u \in \mathscr{M}_{\Omega}$ telles que les $\lambda u$ sont $\varphi$-intégrables, c'est-à-dire

$$
\int_{\Omega} \varphi(\lambda|u(x)|, x) d \mu<+\infty
$$

pour tout réel $\lambda$ strictement positif, muni de la topologie induite par celle de $L^{\varphi}(\Omega)$. Nous le notons ici $L_{0}^{\varphi}(\Omega)$. L'ensemble des combinaisons linéaires finies des fonctions caractéristiques des ensembles mesurables de mesures finies (autrement appelé ensemble des fonctions simples) appartenant à $L_{0}^{\varphi}(\Omega)$ est dense dans cet espace pour la topologie de $L^{\varphi}(\Omega)$. Cette propriété se démontre exactement comme dans le cas des espaces d'Orlicz.

Remarque 3 . Si $\varphi(t, x)$ est une fonction de $t$ sous-additive pour tout $x$ dans $\Omega$, ce qui est le cas lorsque $\varphi(t, x)$ est une fonction de $t$ concave pour tout $x$ dans $\Omega$, alors on a $L_{0}^{\varphi}(\Omega)=L^{\varphi}(\Omega)$. Rappelons que pour les espaces d'Orlicz $L^{\ell}(\Omega)$, on a aussi $L_{0}(\Omega)=\mathscr{L}^{\varrho}(\Omega)$ si $\varrho$ vérifie la condition $\Delta_{2}$ (cf. [10]).

Définition 5. Dans le cas où $\Omega$ est l'ensemble $\boldsymbol{N}$ des entiers naturels muni de la mesure cardinale, l'espace d'Orlicz engendré par une fonction d'Orlicz $\varrho$ se définit par

$$
l^{\varrho}=\left\{\left(\lambda_{n}\right)_{n \in N} \in K^{N}: \exists \varepsilon>0, \sum_{n=0}^{\infty} \varrho\left(\varepsilon\left|\lambda_{n}\right|\right)<+\infty\right\}
$$


où $\boldsymbol{K}$ est le champ des scalaires réels ou complexes. Cet espace se réduit à

$$
l^{e}=\left\{\left(\lambda_{n}\right)_{n \in N} \in K^{N}: \sum_{n=0}^{\infty} \varrho\left(\left|\lambda_{n}\right|\right)<+\infty\right\}
$$

lorsque $\varrho$ vérifie la condition $\Delta_{2}$.

DÉfinition 6. A la fonction d'Orlicz $\varrho$, on peut associer la fonction $\varrho_{(-1)}$ définie par

$$
\varrho_{(-1)}(t)= \begin{cases}0 & \text { si } t=0, \\ 1 / \varrho\left(t^{-1}\right) & \text { si } t>0 .\end{cases}
$$

$\varrho_{(-1)}$ est une fonction croissante de $[0,+\infty[$ dans $[0,+\infty[$, de plus elle vérifie la condition $\Delta_{2}$ s'il en est ainsi de la fonction $\varrho$.

Notation. Si $B$ est une partie d'un espace vectoriel et $\lambda=\left(\lambda_{n}\right)_{n \in N}$ une suite de scalaires, nous pouvons poser

$$
\sum_{n \geqslant 0}^{\prime} \lambda_{n} B=\bigcup_{N \geqslant 0} \sum_{n=0}^{N} \lambda_{n} B
$$

La partie (a) de la proposition 2 dans [13] (cf. [14], corollaire 3.4.2) peut être modifiée comme suit, en supprimant la condition $\Delta_{2}$ faite sur la fonction d'Orlicz:

Proposition 1.1. Étant donnés un espace mesuré $\Omega$ de mesure sans atome, une fonction d'Orlicz $\varrho$ et une suite $\left(\lambda_{n}\right)_{n \in N}$ de scalaires, si $\left(\lambda_{n}\right)_{n \in N} \notin \rho^{(-1)}$, alors pour tout $\varepsilon>0$ on $a$

$$
\sum_{n \geqslant 0}^{\prime} \lambda_{n} \varepsilon B_{\Omega}^{Q}(\varepsilon) \cap L_{0}(\Omega)=L_{0}(\Omega) .
$$

Sachant que l'ensemble des $U_{\varepsilon}=\sum_{n \geqslant 0}^{\prime} \lambda_{n} \varepsilon B_{\Omega}^{e}(\varepsilon), \dot{\varepsilon}>0$, est un système fondamental de voisinages de 0 pour une topologie vectorielle sur $L(\Omega)$ (cf. (1)) moins fine que la topologie de la $F$-norme $v_{Q}$ associée à $\varrho$ (cf. (2)) et en tenant compte de la remarque 2 , on démontre exactement comme dans [13] que l'adhérence de 0 dans cette topologie, soit l'ensemble $\bigcap_{\varepsilon>0} \sum_{n \geqslant 0}^{\prime} \lambda_{n} \varepsilon B_{\Omega}^{e}(\varepsilon)$, contient l'ensemble des fonctions caractéristiques appartenant à $L_{0}(\Omega)$ des sous-ensembles mesurables de $\Omega$.

Remarque 4. Grâce à cette propriété, la condition nécessaire d'existence d'une application linéaire continue non nulle de $L^{\mathscr{Q}_{0}}(\Omega)$ dans $L^{\mathscr{1}}(\Omega)$ démontrée par $\mathrm{Ph}$. Turpin (cf. [14], théorème 3.4.8), avec $\varrho_{1}$ concave et $\varrho_{0}$ vérifiant la condition $\Delta_{2}$, est préservée si on abandonne cette dernière condition et qu'à la place de $L^{\ell 0}(\Omega)$ on envisage plutôt $L_{0}(\Omega)$ où $\varrho$ est une fonction d'Orlicz quelconque.

Notation. Pour tout sous-ensemble mesurable $A \subset \Omega$, la fonction caractéristique de $A$ sera notée $1_{\mathcal{A}}$ tandis que le sous-espace de $L_{0}^{\varphi}(\Omega)$ formé de (classes de) fonctions mesurables appartenant à $L_{0}^{\varphi}(\Omega)$ mais presque partout nulles sur $\Omega \backslash A$ sera désigné par $L_{0}^{\varphi}(A)$.

Défintrion 7. Si $\varphi$ est une fonction de Musielak-Orlicz sur $\Omega$, on dit que $x$ (ou $\varphi_{x}$ ) est dominé par $x_{0}$ (ou par $\varphi_{x_{0}}$ ) lorsque $\limsup \varphi(t, x) / \varphi\left(t, x_{0}\right)$ $<+\infty$ et on note cela par $x \prec x_{0}$ ou $\varphi_{x} \prec \varphi_{x_{0}}$.

Cette définition permet d'envisager une relation de préordre sur l'espace mesuré $\Omega$ ou sur l'ensemble des fonctions d'Orlicz $\varphi_{x}, x \in \Omega$.

Définition 8. Étant donné une fonction de Musielak-Orlicz $\varphi$ sur un espace mesuré $\Omega$, nous appelons les ensembles $\left\{x \in \Omega: \varphi_{x} \prec \varphi_{x_{0}}\right\}$ et $\left\{x \in \Omega: \varphi_{x_{0}} \prec \varphi_{x}\right\}$ la section commençante et section finissante de borne $x_{0}$ respectivement.

Ces ensembles sont mesurables car la fonction $g(x, y)=\limsup \varphi(t, y)$ $\times \varphi(t, x)^{-1}$ est mesurable par rapport à chaque variable séparément.

Définition 9. Une fonction de Musielak-Orlicz $\varphi$ sur un espace mesuré $\Omega$ vérifie la condition $\mathrm{C}_{0}$ (respectivement la condition $\mathrm{C}_{1}$ ) lorsque pour tout sous-ensemble mesurable $A$ dans $\Omega$ de mesure non nulle, il existe un point $x_{A}$ de $A$ tel que la trace sur $A$ de la section commençante de borne $x_{A}$ (respectivement la trace sur $A$ de la section finissante de borne $x_{A}$ ) est de mesure non nulle.

Exemple 1. Soit $\varphi$ la fonction de Musielak-Orlicz $\varphi(t, x)=t^{x}$ pour $x$ appartenant à $\Omega=] 0,1]$ et pour $0 \leqslant t$. C'est une fonction de $t$ concave pour tout $x$ fixé dans $] 0,1]$. Pour tout $x$ dans ]0,1], les sections commençantes de borne $x$ sont du type $] 0, x]$ tandis que les sections finissantes de borne $x$ s'écrivent $[x, 1]$. Les conditions $C_{0}$ et $C_{1}$ sont trivialement vérifiées par cette fonction.

EXEMPLE 2. De manière plus générale, on peut considérer une fonction monotone croissante $p$ définie sur $\Omega=\boldsymbol{R}$ et à valeurs dans $] 0,1]$ et envisager la fonction définie sur $\left[0, \infty\left[\times \boldsymbol{R}\right.\right.$ par $\varphi(t, x)=t^{p(x)}$. C'est de nouveau une fonction de Musielak-Orlicz telle que pour chaque $x$ fixé dans $R, \varphi(t, x)$ est une fonction de $t$ concave. Comme $] 0,1]$ est un ensemble totalement ordonné par la relation usuelle d'inégalité, toutes les fonctions d'Orlicz $\varphi_{x}$, $x \in \boldsymbol{R}$, associées à cette fonction sont comparables par la relation définie précédemment. Les sections commençantes et finissantes s'écrivent respectivement $]-\infty, x]$ et $[x,+\infty[$ pour tout $x$ dans $\boldsymbol{R}$ et il est tout aussi évident que les conditions $\mathrm{C}_{0}$ et $\mathrm{C}_{1}$ sont satisfaites par la fonction $\varphi$.

Définition 10. Un ensemble mesurable $H$ dans $\Omega$ est dit héréditaire lorsque quel que soit $y$ dans $H$, la section commençante de borne $y$ est entièrement contenue dans $H$.

Les sections commençantes dans $\Omega$ sont naturellement des sousensembles héréditaires de $\boldsymbol{\Omega}$. 
Notation. $L_{0}^{\varphi_{0}^{0}}\left(\Omega_{0}\right)$ et $L_{0}^{\varphi^{1}}\left(\Omega_{1}\right)$ étant deux espaces de Musielak-Orlicz, l'ensemble des opérateurs linéaires continus de $L_{0}^{0}\left(\Omega_{0}\right)$ dans $L_{0}^{\varphi^{1}}\left(\Omega_{1}\right)$ sera noté $\mathscr{L}\left[L_{0}^{\varphi^{0}}\left(\Omega_{0}\right), L_{0}^{\varphi^{1}}\left(\Omega_{1}\right)\right]$ tandis que $\mathscr{L}\left[L_{0}^{\varphi}(\Omega)\right]$ désignera l'algèbre des endomorphismes continus de $L_{0}^{p}(\Omega)$.

Définition 11. Nous dirons d'un espace de Musielak-Orlicz $L_{0}^{\varphi}(\Omega)$ qu'il est transitif lorsque les seuls sous-espaces fermés invariants par rapport à tous les endomorphismes continus de $L_{0}^{\varphi}(\Omega)$ sont les sous-espaces triviaux. Dans le cas contraire, l'espace sera dit intransitif.

\section{$\$ 2$ Opérateurs linéaires continus entre espaces de Musielak-Orlicz non} localement convexes. Lorsque $\varphi$ et $\varrho$ sont respectivement une fonction de Musielak-Orlicz et une fonction d'Orlicz sur $\Omega$, nous pouvons poser:

$$
\begin{gathered}
1 \vee \varphi_{x}(t)=\sup \{1, \varphi(t, x)\} \\
U_{Q, h}^{\varphi}=\left\{x \in \Omega: \sup _{t>0} \varphi(t, x) / 1 \vee \varrho(t) \leqslant h\right\}, \quad h \in N_{0}, \\
V_{\varphi, h}^{e}=\left\{x \in \Omega: \sup _{t>0} \varrho(t) / 1 \vee \varphi_{x}(t) \leqslant h\right\}
\end{gathered}
$$

où $N_{0}=N \backslash\{0\}$.

LEMME 2.1. Soient $\Omega$ un espace mesuré de mesure bornée $\mu$ sans atome et $L_{0}^{e}(\Omega)$ et $L_{0}^{\varphi}(\Omega)$ des espaces respectivement d'Orlicz et de Musielak-Orlicz.

(i) Si $\varphi_{x}$ est dominé par @ pour presque tous les $x$ dans $\Omega$, alors l'union de tous les $L_{0}^{Q}\left(U_{Q, h}^{\varphi}\right)$ lorsque $h$ varie dans $N_{0}$ est dense dans $L_{0}^{\varphi}(\Omega)$.

(ii) Si $\varrho$ est dominé par $\varphi_{x}$ pour presque tous les $x$ dans $\Omega$, alors $L_{0}^{\varphi}(\Omega)$ s'injecte continûment dans le produit topologique de tous les $L_{0}^{\ell}\left(V_{\varphi, h}^{e}\right)$ lorsque $h$ varie dans $\boldsymbol{N}_{0}$

Démonstration. (i) Nous savons que les conditions

$$
\begin{gathered}
\limsup _{t \rightarrow \infty} \frac{\varphi(t, x)}{\varrho(t)}<+\infty \quad \text { et } \\
\sup _{t>0} \frac{\varphi(t, x)}{1 \vee \varrho(t)}<+\infty
\end{gathered}
$$

sont équivalentes, donc on peut écrire

$$
\mu(\Omega)=\mu\left(\left\{x \in \Omega: \varphi_{x} \prec \varrho\right\}\right)=\mu\left(\bigcup_{h \in N_{0}} U_{\varrho, h}^{\varphi}\right),
$$

où la suite $\left(U_{Q, h}^{\varphi}\right)_{h \in N_{0}}$ est croissante par inclusion. Par conséquent, les ensembles $\bigcup_{h \in N_{0}} L_{0}^{Q}\left(U_{Q, h}^{\varphi}\right)$ et $\bigcup_{h \in N_{0}} L_{0}^{\varphi}\left(U_{Q, h}^{\varphi}\right)$ sont des sous-espaces respectivement de $L_{0}(\Omega)$ et $L_{0}^{\varphi_{0}}(\Omega)$. Puisque pour tout nombre naturel $h \geqslant 1$ on a

$$
\varphi(t, x) \leqslant h(1+\varrho(t)) \quad \forall x \in U_{\varrho, h}^{\varphi},
$$

le théorème d'inclusion des espaces de Musielak-Orlicz (cf. [2], p. 879) implique que $L_{0}\left(U_{e, h}^{\varphi}\right) \subset L_{0}^{\varphi}\left(U_{e, h}^{\varphi}\right)$ pour tout $h$ dans $N_{0}$ et par conséquent nous obtenons aussi

$$
\bigcup_{h \in N_{0}} L_{0}^{Q}\left(U_{Q, h}^{\varphi}\right) \subset \bigcup_{h \in N_{0}} L_{0}^{\varphi}\left(U_{Q, h}^{\varphi}\right)
$$

Il est évident que $\bigcup_{h \in N_{0}} L_{0}^{\varphi}\left(U_{Q, h}^{\varphi}\right)$ est dense dans $L_{0}^{\varphi}(\Omega)$. Alors, pour prouver (i), il suffit de montrer que l'inclusion précédente est dense.

En effet, pour tout $u$ dans le sous-espace $\bigcup L_{0}^{\varphi}\left(U_{Q, h}^{\varphi}\right)$, il existe un nombre naturel $h_{u} \geqslant 1$ tel que $u$ soit un élément de $L_{0}^{\varphi}\left(U_{Q, h}^{\varphi}\right)$. La mesure étant finie, il est clair que toutes les fonctions simples (combinaisons linéaires finies des fonctions caractéristiques des ensembles mesurables) appartenant à $L_{0}^{\varphi}\left(U_{Q, h_{y}}^{\varphi}\right)$ appartiennent aussi à $L_{0}^{Q_{0}}\left(U_{Q, h_{u}}^{\varphi}\right)$. Ainsi, en vertu de la remarque 2, l'inclusion $L_{0}\left(U_{Q, h_{h}}^{\varphi}\right) \subset L_{0}^{\varphi}\left(U_{Q, h_{u}}^{\varphi}\right)$ est dense pour la topologie de $L_{0}^{\varphi}(\Omega)$. Par conséquent, pour tout $\varepsilon>0$, il existe un $u_{\varepsilon} \in L_{0}^{Q}\left(U_{Q, h_{u}}^{\varphi}\right)$ tel que $v_{\varphi}\left(u-u_{\varepsilon}\right)<\varepsilon$, ce qui en vertu de (2) établit l'assertion (i).

(ii) En vertu de l'équivalence entre les conditions (12) et (13), nous avons aussi

$$
\mu(\Omega)=\mu\left(\left\{x \in \Omega: \varrho \prec \varphi_{x}\right\}\right)=\mu\left(\bigcup_{h \in N_{0}} V_{\varphi, h}^{Q}\right) .
$$

Sur chaque $V_{\varphi, h}^{e}$ linégalité suivante est vérifiée:

$$
\varrho(t) \leqslant h(1+\varphi(t, x)) \quad \forall x \in V_{\varphi, h}^{e},
$$

qui nous permet d'appliquer à nouveau le théorème d'inclusion des espaces de Musielak-Orlicz à chaque $V_{\varphi, h}^{e}$ pour obtenir l'inclusion continue $L_{0}^{\varphi}\left(V_{\varphi, h}^{Q}\right) \subset L_{0}^{Q}\left(V_{\varphi, h}^{Q}\right)$ pour tout $h$ naturel $\geqslant 1$.

Soit $p_{h}$ l'opérateur de projection de $L_{0}^{\varphi}(\Omega)$ sur $L_{0}^{\varphi}\left(V_{\varphi, h}^{Q}\right)$ pour chaque $h$ naturel $\geqslant 1$ défini par

$$
p_{h}(u)=u \cdot 1_{V_{\varphi, h}^{e}}=u_{h}
$$

où la multiplication est à comprendre dans le sens suivant:

$$
\left(u \cdot 1_{V_{\varphi, h}^{e}}\right)(x)=u(x) \cdot 1_{V_{\varphi, h}^{e}}(x),
$$

où $1_{V_{\varphi, h}^{e}}$ est la fonction caractéristique de $V_{\varphi, h}^{e}$. Posons à présent $p=\prod_{h \in N_{0}} p_{h}$. Alors limage que $p$ prend sur un élément quelconque $u$ de $L_{0}^{\varphi}(\Omega)$ se définit comme suit:

$$
p(u)=\prod_{h \in N_{0}} p_{h}(u)=\left(u_{h}\right)_{h \in N_{0}} .
$$


L'application $p$ ainsi définie est linéaire et injective de $L_{0}^{\varphi}(\Omega)$ dans le produit topologique $\prod L_{0}^{\varphi}\left(V_{\varphi, h}^{Q}\right)$. Compte tenu de la continuité des $p_{h}, p$ est aussi continu par définition d'une topologie produit. Donc, si $I$ désigne linclusion continue

$$
\prod_{h \in N_{0}} L_{0}^{\varphi}\left(V_{\varphi, h}^{\ell}\right) \subset \prod_{h \in N_{0}} L_{0}^{\ell_{0}}\left(V_{\varphi, h}^{\ell}\right)
$$

Iop est une application linéaire continue injective de $L_{0}^{\varphi}(\Omega)$ dans le produit topologique $\prod_{h \in N_{0}} L_{0}^{e_{0}}\left(V_{\varphi, h}^{e}\right)$.

LEMME 2.2. Si $\varphi$ est une fonction de Musielak-Orlicz sur un espace mesuré $\Omega$ de mesure finie sans atome, vérifiant la condition $\mathrm{C}_{0}$ (respectivement la condition $\left.C_{1}\right)$, alors il existe une suite $\left(x_{n}\right)_{n \in N}$ d'éléments de $\Omega$ telle que $\Omega$ et $\bigcup_{n \in N}\left\{x \in \Omega: \varphi_{x} \prec \varphi_{x_{n}}\right\}$ (respectivement $\Omega$ et $\left.\bigcup_{n \in \mathcal{N}}\left\{x \in \Omega: \varphi_{x_{n}} \prec \varphi_{x}\right\}\right)$ soricnt de même mesure.

Démonstration. Soit $\mathfrak{A}$ l'ensemble des familles finies ou dénombrables $\left(A_{i}\right)_{i \in I}$ où les $A_{i}$ sont des ensembles mesurables de mesures non nulles et deux à deux disjoints, chaque $A_{i}$ étant contenu dans une section commençante. La condition $\mathrm{C}_{0}$ sur $\varphi$ permet de construire facilement une telle famille à partir de la trace sur un ensemble mesurable de mesure non nulle et cela par les sections commençantes.

Nous pouvons définir sur $\mathfrak{A}$ une relation d'ordre comme suit: $\forall \mathscr{M}_{0}, \mathscr{M}_{1} \in \mathfrak{Q}: \mathscr{M}_{0} \leqslant \mathscr{M}_{1}$ si les éléments de $\mathscr{M}_{0}$ sont dans $\mathscr{M}_{1}$.

$\mathfrak{Q}$ ainsi ordonné est un inductif. En effet, soit $\mathfrak{F}$ une partie quelconque totalement ordonnée dans $\mathfrak{A}$. La réunion de toutes les familles appartenant à $\mathfrak{F}$ de sous-ensembles mesurables de mesures non nulles et deux à deux disjoints est un majorant de $\mathfrak{F}$ pour la relation d'ordre définie ci-dessus. Ce majorant appartient à $\mathscr{2}$, car la mesure $\mu$ sur $\Omega$ est bornée. Alors en vertu de l'axiome de Zorn, il existe au moins une famille maximale $\mathscr{M} \in \mathfrak{\mathscr { M }}$ avec $\mathscr{M}$ $=\left(M_{n}\right)_{n \in N}$ telle que $\mu\left(\Omega \backslash \bigcup_{n \in N} M_{n}\right)=0$, car sinon, compte tenu de la condition $\mathrm{C}_{0}, \Omega \backslash \bigcup_{n \in N} M_{n}$ contiendrait un élément $y$ tel que l'ensemble

$$
\left(\Omega \backslash \bigcup_{n \in N} M_{n}\right) \cap\left\{x \in \Omega: \varphi_{x} \prec \varphi_{y}\right\}=A
$$

soit de mesure non nulle. $\mathscr{M} \cup\{A\}$ serait une famille de $\mathscr{A}$ contenant $\mathscr{M}$ et $\mathscr{H}$ ne serait pas maximal. Par conséquent, en vertu de la définition des familles de $\mathscr{A}$, à $\mathscr{M}=\left(M_{n}\right)_{n \in N}$ nous pouvons associer une suite des sections commençantes $\left(\left\{x \in \Omega: \varphi_{x} \prec \varphi_{x_{n}}\right\}\right)_{n \in N}$ telle que $M_{n} \subset\left\{x \in \Omega: \varphi_{x} \prec \varphi_{x_{n}}\right\}$ pour chaque $n \in N$ et $\mu\left(\Omega \backslash \bigcup_{n \in N}\left\{x \in \Omega: \varphi_{x} \prec \varphi_{x_{n}}\right\}\right)=0$.

Lorsque la condition $\mathrm{C}_{1}$ sur $\varphi$ est satisfaite, on montre de manière similaire l'existence d'une suite d'éléments $\left(y_{m}\right)_{m \in N}$ de $\Omega$ telle que $\mu\left(\Omega \backslash \bigcup_{m \in N}\left\{x \in \Omega: \varphi_{y_{m}} \prec \varphi_{x}\right\}\right)=0$.

THÉORÈme 2.3. Soient $\Omega_{0}$ et $\Omega_{1}$ deux espaces mesurés de mesures respectives $\mu_{0}$ et $\mu_{1} \sigma$-finies sans atome et $\varphi^{0}, \varphi^{1}$ deux fonctions de Musielak-Orlicz sur $\Omega_{0}$ et $\Omega_{1}$ respectivement, $\varphi^{0}$ vérifiant la condition $\mathrm{C}_{0}, \varphi^{1}$ satisfaisant à la condition $\mathrm{C}_{1}$ et de plus telle que pour chaque $x$ fixé dans $\Omega_{1}, \varphi^{1}(t, x)$ est une fonction de $t$ concave. Si pour tout couple $(x, y)$ dans $\Omega_{0} \times \Omega_{1}$ on a

$$
\limsup _{t \rightarrow \infty} \frac{\varphi^{1}(t, y)}{\varphi^{0}(t, x)}=+\infty,
$$

alors tout opérateur linéaire continu de $L_{0}^{\varphi_{0}^{0}}\left(\Omega_{0}\right)$ dans $L_{0}^{\varphi^{1}}\left(\Omega_{1}\right)$ est nul.

Démonstration. Puisque les mesures $\mu_{0}$ et $\mu_{1}$ sont $\sigma$-finies, nous avons

$$
\begin{array}{lll}
\Omega_{0}=\bigcup_{h \in N} \Omega_{h}^{0}, & \mu\left(\Omega_{h}^{0}\right)<+\infty & \forall h \in N, \\
\Omega_{1}=\bigcup_{k \in N} \Omega_{k}^{1}, & \mu\left(\Omega_{k}^{1}\right)<+\infty & \forall k \in N .
\end{array}
$$

(i) Montrons d'abord que tout opérateur linéaire continu de $L_{0}^{\varphi^{0}}\left(\Omega_{h}^{0}\right)$ dans $L_{0}^{\varphi}\left(\Omega_{k}^{1}\right)$ est nul pour tout couple $(h, k) \in \boldsymbol{N} \times \boldsymbol{N}$. Soit donc $(h, k)$ un couple quelconque de nombres naturels. Puisque les conditions $C_{0}$ et $C_{1}$ sont vérifiées respectivement par $\varphi^{0}$ et $\varphi^{1}$, en vertu du lemme 2.2 il existe une suite $\left(x_{n}\right)_{n \in N}$ d'éléments de $\Omega_{h}^{0}$ et une suite $\left(y_{m}\right)_{m \in N}$ d'éléments de $\Omega_{k}^{1}$ telles que

$$
\begin{aligned}
& \mu_{0}\left(\Omega_{h}^{0}\right)=\mu_{0}\left(\bigcup_{n \in \mathbb{N}}\left\{x \in \Omega_{h}^{0}: \varphi_{x}^{0} \prec \varphi_{x_{n}}^{0}\right\}\right), \\
& \mu_{1}\left(\Omega_{k}^{1}\right)=\mu_{1}\left(\bigcup_{m \in \mathbb{N}}\left\{y \in \Omega_{k}^{1}: \varphi_{y_{m}}^{1} \prec \varphi_{y}^{1}\right\}\right) .
\end{aligned}
$$

Compte tenu de l'équivalence entre les conditions (12) et (13), on sait que pour tout $m$ naturel on a

$$
\left\{y \in \Omega_{k}^{1}: \varphi_{y_{m}}^{1} \prec \varphi_{y}^{1}\right\}=\bigcup_{r \in N_{0}} V_{\varphi^{1, r}, \varphi_{m}^{1}}^{\varphi_{1}^{1}} \quad \text { (cf. (11)). }
$$

Alors, en vertu de la partie (ii) du lemme 2.1 il existe une injection continue notée $p$ de $L_{0}^{\varphi^{1}}\left(\Omega_{k}^{1}\right)$ dans le produit topologique des $L_{0}^{\varphi^{1}}\left(V_{\varphi^{1}, r}^{\varphi_{m}^{1}}\right)$ pour $(m, r)$
variant dans $N \times N_{0}$, définie d'après (16) et (17) par

$$
p(u)=\left(u_{m r}\right)_{(m, r) \in N \times N_{0}} \quad \forall u \in L_{0}^{\varphi^{1}}\left(\Omega_{k}^{1}\right),
$$

où $u_{m r} \in L_{0}^{\varphi_{y_{m}}^{1}}\left(V_{\varphi, r, r}^{\varphi_{y_{m}}^{1}}\right)$ pour chaque couple $(m, r) \in \boldsymbol{N} \times \boldsymbol{N}_{0}$. 
D'autre part, pour un $n \in \boldsymbol{N}$ quelconque, la section commençante $\left\{x \in \Omega_{h}^{0}: \varphi_{x}^{0} \prec \varphi_{x_{n}}^{0}\right\}$ est constituée des éléments $x \in \Omega_{h}^{0}$ dominés par $x_{n}$ et en vertu de la partie (i) du lemme 2.1,

$$
\bigcup_{s \in N_{0}} L_{0}^{\varphi x_{x_{n}}}\left(U_{\varphi_{x_{n}}^{0}, s}^{\varphi}\right)
$$

est dense dans $L_{0}^{\varphi^{0}}\left(\left\{x \in \Omega_{h}^{0}: \varphi_{x}^{0} \prec \varphi_{x_{n}}^{0}\right\}\right)$. Dans ce cas, pour tout opérateur linéaire continu $T$ de ce dernier espace dans $L_{0}^{\varphi_{0}^{1}}\left(\Omega_{k}^{1}\right)$, le composé poT est linéaire continu de $L_{0}^{\varphi_{0}^{0}}\left(\left\{x \in \Omega_{h}^{0}: \varphi_{x}^{0} \prec \varphi_{x_{n}}^{0}\right\}\right)$ dans

$$
\prod_{\substack{m \in N_{0} \\ r \in N_{0}}} L_{0}^{\varphi_{y_{m}}^{1}}\left(V_{\varphi^{1}, r}^{\varphi_{y_{m}}^{1}}\right)
$$

telle que

$$
p \circ T(u)=\left(w_{m r}\right)_{(m, r) \in N \times N_{0}} \quad \forall u \in \bigcup_{s \in N_{0}} L_{0}^{\varphi x_{n}}\left(U_{\varphi_{x_{n}}^{0}}^{\varphi^{0}}\right)
$$

où $w_{m r} \in L_{0}^{\varphi_{0}^{1}} y_{m}^{1}\left(V_{\varphi^{1}, r}^{\varphi_{y_{m}}^{1}}\right)$ pour chaque $(\dot{m}, r) \in N \times N_{0}$.

Mais par hypothèse, $\limsup \varphi^{1}\left(t, y_{m}\right) / \varphi^{0}\left(t, x_{n}\right)=+\infty \forall(m, n) \in N \times N$,

alors en vertu de la remarque 4 , la condition nécessaire d'existence d'un opérateur linéaire continu non nul entre des espaces d'Orlicz (cf. [14], théorème 3.4.8) est applicable et on obtient $w_{m r}=0$ pour tout couple $(m, r)$ dans $N \times N_{0}$. Comme $p$ est une injection, on en déduit que $T$ est nul sur

$$
\bigcup_{s \in N_{0}} L_{0}^{\varphi x_{n}}\left(U_{\varphi_{x_{n}}^{0}, \varphi^{0}}^{\varphi_{0}^{0}}\right)
$$

qui est un sous-espace dense dans $L_{0}^{\varphi^{0}}\left(\left\{x \in \Omega_{h}^{0}: \varphi_{x}^{0} \prec \varphi_{x_{n}}^{0}\right\}\right)$. Par conséquent $T$ est nul sur ce dernier espace et on en conclut que

$$
\mathscr{L}\left[L_{0}^{\varphi_{0}^{0}}\left(\left\{x \in \Omega_{h}^{0}: \varphi_{x}^{0} \prec \varphi_{x_{n}}^{0}\right\}\right), L_{0}^{\varphi_{0}^{1}}\left(\Omega_{k}^{1}\right)\right]=\{0\} .
$$

L'égalité ci-dessus est valable quel que soit le 3 -uple $(h, n, k)$ dans $\boldsymbol{N} \times \boldsymbol{N} \times \mathbf{N}$.

Posons à présent

$$
D_{m}=\bigcup_{n \leqslant m}\left\{x \in \Omega_{h}^{0}: \varphi_{x}^{0} \prec \varphi_{x_{n}}^{0}\right\}
$$

Puisque $D_{0}=\left\{x \in \Omega_{h}^{0}: \varphi_{x}^{0} \prec \varphi_{x_{0}}^{0}\right\}$, on démontre par récurrence que $\mathscr{L}\left[\mathscr{L}_{0}^{0}\left(D_{m}\right), L_{0}^{\varphi}\left(\Omega_{k}^{1}\right)\right]=\{0\}$ pour tout $(m, k) \in N \times N$.

Or on sait que $\Omega_{h}^{0}=\bigcup_{m \in N} D_{m}$ pour un naturel $h$ quelconque, la suite $\left(D_{m}\right)_{m \in N}$ étant croissante; l'ensemble $\bigcup_{m \in N} L_{0}^{\varphi_{0}^{0}}\left(D_{m}\right)$ est donc un sous-espace dense de $L_{0}^{\varphi^{0}}\left(\Omega_{h}^{0}\right)$. Par conséquent on a

$$
\mathscr{L}\left[L_{0}^{\varphi_{0}^{0}}\left(\Omega_{h}^{0}\right), L_{0}^{\varphi_{0}^{1}}\left(\Omega_{k}^{1}\right)\right]=\{0\} \quad \forall(h, k) \in N \times N .
$$

En posant encore $H_{m}=\bigcup_{h \leqslant m} \Omega_{h}^{0}$ avec $H_{0}=\Omega_{0}^{0}$ et sachant que $\left(H_{m}\right)_{m \in \mathbf{N}}$ est une suite croissante de sous-ensembles mesurables de $\Omega_{0}$ telle que $\Omega_{0}$ $=\bigcup_{m \in N} H_{m}$, on montre par récurrence comme précédemment que

$$
\mathscr{L}\left[L_{0}^{\varphi_{0}^{0}}\left(\Omega_{0}\right), L_{0}^{\varphi^{1}}\left(\Omega_{k}^{1}\right)\right]=\{0\} \quad \forall k \in N .
$$

Soit à présent $p^{\prime}$ l'application de $L_{0}^{\varphi^{1}}\left(\Omega_{1}\right)$ dans le produit topologique $\prod_{k \in N} L_{0}^{p^{1}}\left(\Omega_{k}^{1}\right)$ définie d'après (19), injective et continue. Puisque le composé avec $p^{\prime}$, soit $p^{\prime} \circ T$, de tout opérateur linéaire continu $T$ de $L_{0}^{\varphi^{0}}\left(\Omega_{0}\right)$ dans $L_{0}^{\varphi^{1}}\left(\Omega_{1}\right)$ est nulle en vertu de (21), $T$ lui-même est nul.

3. Sous-espaces fermés non triviaux invariants dans les espaces de MusielakOrlicz non localement convexes. Les espaces de Musielak-Orlicz engendrés par des fonctions $\varphi$ telles que $\varphi(t, x)$ est une fonction de $t$ concave pour tout $x$ appartenant à un certain espace mesuré $\Omega$ fournissent des exemples d'espaces vectoriels topologiques généralement non localement convexes. C'est dans ces espaces, lorsque $\Omega$ est un espace métrique complet séparable muni d'une meșure bornée non atomique et régulière, que nous caractérisons les sous-espaces fermés non triviaux invariants par rapport à tous les endomorphismes continus de ces espaces.

ThÉORÈme 3.1. Étant donnés deux espaces de Musielak-Orlicz $L_{0}^{\varphi^{0}}\left(\Omega_{0}\right)$ et $\underline{L}_{0}^{\varphi^{1}}\left(\Omega_{1}\right)$ où $\Omega_{0}$ et $\Omega_{1}$ sont des espaces métriques complets séparables munis des mesures respectives $\mu_{0}, \mu_{1}$ bornées, régulières et sans atome, si

$$
\limsup _{t \rightarrow \infty} \frac{\varphi^{1}(t, y)}{\varphi^{0}(t, x)}<+\infty
$$

pour tout couple $(x, y)$ dans $\Omega_{0} \times \Omega_{1}$, alors quel que soit lélément non nul u dans $L_{0}^{\varphi^{0}}\left(\Omega_{0}\right)$, lensemble des images $T u$ où $T$ parcourt $\mathscr{L}\left[L_{0}^{\varphi^{0}}\left(\Omega_{0}\right), L_{0}^{\varphi^{1}}\left(\Omega_{1}\right)\right]$ est dense dans $L_{0}^{\varphi} 1\left(\Omega_{1}\right)$.

Démonstration. Soit $u_{1} \neq 0$ un élément quelconque dans $L_{0}^{\varphi^{0}}\left(\Omega_{0}\right)$ et considérons l'ensemble mesurable

$$
S_{u_{1}, \varepsilon}=\left\{x \in \Omega_{0}:\left|u_{1}(x)\right|>\varepsilon\right\}
$$

où $\varepsilon>0$ est tel que $S_{u_{1}, \varepsilon}$ soit de mesure non nulle. $\mu_{0}$ étant une mesure régulière, il existe un compact $C \subset S_{u_{1}, e}$ de mesure non nulle tel que la mesure de $S_{u_{1}, \varepsilon} \backslash C$ soit arbitrairement petite.

Prenons un ensemble mesurable $A \subset \Omega_{1}$ quelconque de mesure non nulle tel que sa fonction caractéristique $1_{A}$ soit un élément de $L_{0}^{1}\left(\Omega_{1}\right)$ Puisque la mesure $\mu_{1}$ est également régulière, il existe un nombre naturel $m_{0}$ $\geqslant 1$ tel qu'à tout autre nombre naturel $m \geqslant m_{0}$ on puisse associer un 
compact $C_{m} \subset A$ de mesure non nulle satisfaisant à la condition

$$
\mu_{1}\left(A \backslash C_{m}\right)<\frac{1}{m}
$$

Pour un nombre naturel $m \geqslant m_{0}$ quelconque mais fixé et pour tout sous-ensemble mesurable $B \subset C_{m}$, nous pouvons poser: $\bar{\mu}_{m}(B)$ $=\mu_{1}(B) / \mu_{1}\left(C_{m}\right)$. De même, pour tout sous-ensemble mesurable $D \subset C$, nous pouvons écrire: $\bar{\mu}(D)=\mu_{0}(D) / \mu_{0}(C) . \bar{\mu}$ et $\bar{\mu}_{m}$ sont des mesures normalisées sans atome respectivement sur $C$ et $C_{m}$. Comme $C$ et $C_{m}$ sont des compacts, en vertu du théorème d'existence d'une équivalence borélienne (cf. [11], Theorem 9 , p. 270) nous pouvons trouver des ensembles mesurables $C^{0} \subset C$ et $C_{m}^{0} \subset C_{m}$ tels que

$$
\bar{\mu}\left(C \backslash C^{0}\right)=0, \quad \bar{\mu}_{m}\left(C_{m} \backslash C_{m}^{0}\right)=0,
$$

ainsi qu'une équivalence borélienne $q$ de $C_{m}^{0}$ sur $C^{0}$ telle que $\bar{\mu}=\bar{\mu}_{m} q^{-1}$. Alors en vertu de la condition (22) on a

$$
\limsup _{t \rightarrow \infty} \frac{\varphi^{1}\left(t, q^{-1}(x)\right)}{\varphi^{0}(t, x)}<+\infty \quad \forall x \in C^{0} .
$$

Posons encore

$$
B_{h}=\left\{x \in C^{0}: \sup _{t>0} \frac{\varphi^{1}\left(t, q^{-1}(x)\right)}{1 \vee \varphi^{0}(t, x)} \leqslant h\right\}, \quad h \in N_{0} .
$$

On a $C^{0}=\bigcup_{h \in N_{0}} B_{h}$. D'autre part,

$$
q^{-1}\left(C^{0}\right)=\bigcup_{h \in N_{0}} q^{-1}\left(B_{h}\right)=C_{m}^{0}
$$

Prenons un $h_{0} \in N_{0}$ suffisamment grand pour que

$$
\bar{\mu}_{m}\left(q^{-1}\left(B_{h}\right)\right)=\bar{\mu}\left(B_{h}\right)>0 \quad \forall h \geqslant h_{0},
$$

la suite $\left(B_{h}\right)_{h \in N_{0}}$ étant croissante. Soit $h \geqslant h_{0}$ un entier quelconque mais fixé et considérons les fonctions $\left(u / u_{1}\right) \cdot 1_{B_{h}}$ pour tout élément $u$ de $I_{0}^{\varphi}\left(\Omega_{0}\right)$. En vertu de (23) et du fait que $u / \varepsilon$ est de nouveau un élément de $L_{0}^{\varphi^{0}}\left(\Omega_{0}\right)$, il est clair que $\left(u / u_{1}\right) \cdot 1_{B_{h}}$ est encore un élément de $L_{0}^{0}\left(B_{h}\right)$ pour tout élément $u$ dans $L_{0}^{\varphi_{0}^{0}}\left(\Omega_{0}\right)$. L'application $T_{h}^{\prime}$ de $L_{0}^{\varphi^{0}}\left(\Omega_{0}\right)$ dans $L_{0}^{\varphi_{0}^{0}}\left(B_{h}\right)$ envoyant $u$ sur $\left(u / u_{1}\right) \cdot 1_{B_{h}}$ est non nulle et linéaire; elle est de plus continue car $\left(1 / u_{1}\right) \cdot 1_{B_{h}}$ est borné. En outre, en appliquant le théorème d'inclusion des espaces de Musielak-Orlicz (cf. [2], p. 879) à $B_{h}$ défini par (26) et le théorème de changement de variables dans une intégrale (cf. [3], Theorem C, p. 163), $(v \circ q) \cdot 1_{q-1_{\left(B_{h}\right)}}$ est un élément de $L_{0}^{\varphi^{1}}\left(\Omega_{1}\right)$ quel que soit $v$ dans $L_{0}^{\varphi^{0}}\left(B_{h}\right)$. L'application $T_{0, h}$ de $L_{0}^{\varphi}\left(B_{h}\right)$ dans $L_{0}^{\varphi^{1}}\left(\Omega_{1}\right)$ envoyant $v$ sur $(v \circ q) \cdot 1_{q^{-1}\left(B_{h}\right)}$ est linéaire continue non nulle pour la même raison que ci-dessus. Posons encore
$T_{h}=T_{0, h} \circ T_{h}^{\prime}$. Nous avons ainsi un nouvel opérateur linéaire continu non nul de $L_{0}^{\varphi_{0}^{0}}\left(\Omega_{0}\right)$ dans $L_{0}^{\varphi^{1}}\left(\Omega_{1}\right)$ et nous obtenons

$$
T_{h}\left(u_{1}\right)=T_{0, h} \circ T_{h}^{\prime}\left(u_{1}\right)=T_{0, h}\left(1_{B_{h}}\right)=\left(1_{B_{h}} \circ q\right) \cdot 1_{q^{-1}\left(B_{h}\right)}=1_{q^{-1}\left(B_{h}\right)} \cdot
$$

Nous en déduisons que $1_{q^{-1}\left(B_{h}\right)}$ appartient à l'ensemble $\left\{T u_{1}: T \in \mathscr{L}\left[L_{0}^{\varphi_{0}^{0}}\left(\Omega_{0}\right)\right.\right.$, $\left.\left.L_{0}^{\varphi^{1}}\left(\Omega_{1}\right)\right]\right\}$ pour tout nombre naturel $h \geqslant h_{0}$. Or $\left(q^{-1}\left(B_{h}\right)\right)_{h \geqslant h_{0}}$ est une suite croissante de sous-ensembles mesurables; donc de (27) il résulte que $1_{c_{m}^{0}}$ appartient à l'adhérence de cet ensemble par rapport à la topologie de $L_{0}^{\varphi}\left(\Omega_{1}\right)$ (cf. (1)). De (25) on conclut qu'il en est de même de $1_{C_{m}}$.

Cependant, $m$ étant un nombre naturel quelconque supérieur ou égal à $m_{0}$, de (24) on déduit que $\left(v_{\varphi^{1}}\left(1_{A}-1_{C_{m}}\right)\right)_{m \in N_{0}}$ est une suite qui converge vers 0 lorsque $m$ tend vers l'infini et de ce fait, $1_{A}$ appartient aussi à l'adhérence de l'ensemble mentionné ci-dessus. Par conséquent, cet adhérence contient l'ensemble total dans $L_{0}^{\varphi}\left(\Omega_{1}\right)$ des fonctions caractéristiques appartenant à $L_{0}^{\varphi^{1}}\left(\Omega_{1}\right)$ des sous-ensembles mesurables de $\Omega_{1}$.

Corollaire 3.2. Soient $\Omega$ un espace métrique complet séparable muni d'une mesure bornée régulière sans atome et $\varphi$ une fonction de Musielak-Orlicz sur $\Omega$. Si

$$
\limsup _{t \rightarrow \infty} \frac{\varphi(t, x)}{\varphi(t, y)}<+\infty
$$

pour tout couple $(x, y)$ dans $\Omega \times \Omega$, alors $L_{0}^{\varphi}(\Omega)$ est transitif.

Démonstration. Soit $\boldsymbol{E}$ un sous-espace fermé quelconque non réduit au singleton nul dans $L_{0}^{\varphi}(\Omega)$ et invariant pour tous les endomorphismes continus de $L_{0}^{\varphi}(\Omega)$. Pour tout élément non nul $u$ de $E$, il est clair qu'on a $\left\{T u: T \in \mathscr{L}\left[L_{0}^{\varphi}(\Omega)\right]\right\} \subset E$ et en vertu du théorème précédent, $E$ est identique à $L_{0}^{\varphi}(\Omega)$. Nous avons donc. prouvé que les seuls sous-espaces fermés de $L_{0}^{\varphi}(\Omega)$ invariants par rapport à tous les endomorphismes continus de cet espace sont les sous-espaces triviaux.

Remarque 5 . On voit en particulier que tout espace d'Orlicz $L_{0}([0,1])$ est transitif au sens de la définition 11. Autrement dit, pour tout élément non nul $u$ de $\mathscr{L}_{0}([0,1])$, l'ensemble $\left\{T u: T \in \mathscr{L}\left[L_{0}([0,1])\right]\right\}$ est dense dans $\mathscr{L}_{0}([0,1])$. On montre dans [4] que cet ensemble est généralement strictement contenu dans $L^{\ell}([0,1])$.

Lemme 3.3. Étant donnés un espace mesuré $\Omega$ de mesure $\mu$ bornée sans atome et une fonction de Musielak-Orlicz $\varphi$ sur $\Omega$, pour tout sous-espace fermé non nul $\boldsymbol{E}$ de $L_{0}^{\varphi}(\Omega)$ invariant pour tous les endomorphismes continus de cet espace, il existe un ensemble mesurable $S_{\mathrm{E}} \subset \Omega$ de mesure non nulle tel que $\boldsymbol{E}$ $=L_{0}^{\varphi}\left(S_{E}\right)$.

Démonstration. Soit $\boldsymbol{E}$ un sous-espace fermé non nul quelconque de $L_{0}^{\varphi}(\Omega)$, invariant pour tous les endomorphismes continus de $L_{0}^{\varphi}(\Omega)$. Montrons 
d'abord que tout élément $u$ de $L_{0}^{\varphi}(\Omega)$ pour lequel il existe une fonction $w$ de $\boldsymbol{E}$ telle que $|u(x)| \leqslant|w(x)|$ pour presque tous les $x$ dans $\Omega$ est aussi un élément de $\boldsymbol{E}$. Soit $u \in L_{0}^{\varphi}(\Omega)$ quelconque vérifiant la propriété ci-dessus et supposons-le différent de la fonction nulle presque partout.

Nous pouvons poser:

$$
S_{u}=\{x \in \Omega: u(x) \neq 0\}, \quad S_{w}=\{x \in \Omega: w(x) \neq 0\} .
$$

Il est évident que ces ensembles sont mesurables et de plus, comme $S_{u} \subset S_{w}, S_{w}$ est de mesure non nulle. Si à tout élément $v$ dans $L_{0}^{\varphi}(\Omega)$ nous associons la fonction $(u / w) \cdot\left(v \cdot 1_{S_{w}}\right)$, celle-ci appartient à $L_{0}^{\varphi}(\Omega)$ car sur $S_{w}$ on a $|u(x) / w(x)| \leqslant 1$; cela permet d'envisager une application $T$ de $L_{0}^{\varphi}(\Omega)$ dans $L_{0}^{\varphi}(\Omega)$ définie par

$$
T v=\frac{u}{w} \cdot\left(v \cdot 1_{S_{w}}\right) \quad \text { pour tout } v \text { dans } L_{0}^{\varphi}(\Omega) .
$$

$T$ est naturellement linéaire et continu. Mais $T w=(u / w) \cdot\left(w \cdot 1_{S_{w}}\right)=u$ et $w$ appartient à $E$; il en résulte que $u$ est également un élément de $E_{w}$ puisque ce dernier est un sous-espace invariant pour $T$. L'assertion est ainsi prouvée.

$\boldsymbol{E}$ étant donc un sous-espace vectoriel solide fermé de $L_{0}^{\varphi}(\Omega)$, on voit comme dans [12], chapitre III, p. 155-156 que $E=L_{0}^{\varphi}\left(S_{E}\right)$ pour quelque ensemble mesurable $S_{E} \subset \Omega$ de mesure non nulle.

Proposition 3.4. Soient donnés un espace métrique complet séparable $\Omega$ muni d'une mesure bornée $\mu$ régulière sans atome et une fonction de MusielakOrlicz $\varphi$ vérifiant les conditions $\mathrm{C}_{0}$ et $\mathrm{C}_{1}$ (cf. Définition 9). Pour tout sousespace fermé $\boldsymbol{E}$ invariant par rapport à tous les endomorphismes continus de $L_{0}^{\varphi}(\Omega)$, il existe $u_{i t}$ sous-ensemble mesurable héréditaire $H \subset \Omega$ tel que $\boldsymbol{E}$ $=L_{0}^{\varphi}(H)$.

Dé monstration. La proposition est triviale si $E=\{0\}$ ou si $E$ $=L_{0}^{\varphi}(\Omega)$. Considérons donc le cas d'un sous-espace fermé non trivial quelconque $\boldsymbol{E}$ de $L_{0}^{\varphi}(\Omega)$, invariant pour tous les endomorphismes continus de cet espace. En vertu du lemme 3.3, il existe un ensemble mesurable $S_{E} \subset \Omega$ de mesure non nulle tel que $E=L_{0}^{\varphi}\left(S_{E}\right)$.

Posons

(28) $H=\left\{y \in \Omega: \exists A_{y} \subset S_{E}\right.$ mesurable, $\left.\mu\left(A_{y}\right)>0, \varphi_{y} \prec \varphi_{x} \forall x \in A_{y}\right\}$.

$H$ est un ensemble mesurable en vertu du théorème de Fubini appliqué à la fonction caractéristique de l'ensemble $\{(x, y) \in \Omega \times \Omega: g(x, y)<+\infty\}$ où $g(x, y)=\limsup _{t \rightarrow \infty} \varphi(t, y) / \varphi(t, x)$. De plus, il est de mesure non nulle. D'autre part, si $y$ est un élément quelconque de $H$, tout $z$ dans $\Omega$ tel que $\varphi_{z} \prec \varphi_{y}$ appartient à $H$, $\operatorname{car} \varphi_{z} \prec \varphi_{y} \prec \varphi_{x}$ pour tout $x$ dans $A_{y}$. $H$ est donc un ensemble héréditaire.

Prouvons que l'ensemble mesurable $S_{E} \backslash H$ est de mesure nulle. Raison- nons par l'absurde. Si $\mu\left(S_{E} \backslash H\right)>0$, prenons $D=S_{E} \backslash H$. Or $\varphi$ vérifie la condition $C_{1}$; il existe donc un $z_{D} \in D$ tel que

$$
\mu\left(D \cap\left\{y \in \Omega: \varphi_{z_{D}} \prec \varphi_{\boldsymbol{y}}\right\}\right)>0 .
$$

Comme $D \cap\left\{y \in \Omega: \varphi_{z_{D}} \prec \varphi_{y}\right\} \subset S_{E}$, il est clair, compte tenu de (28), que $z_{D}$ est un élément de $H$, ce qui est en contradiction avec le fait que $z_{D}$ a été pris dans $S_{E} \backslash H$. Donc $S_{E} \backslash H$ est de mesure nulle. Par conséquent, de

on déduit que

$$
S_{E}=\left(S_{E} \backslash H\right) \cup\left(S_{E} \cap H\right)
$$

$$
E=L_{0}^{\varphi}\left(S_{E}\right)=L_{0}^{\varphi}\left(S_{E} \cap H\right) \subset L_{0}^{\varphi}(H) .
$$

Prouvons l'inclusion inverse. Partons pour cela de $H$ $=\left(H \backslash S_{E}\right) \cup\left(H \cap S_{E}\right)$ et montrons que $H \backslash S_{E}$ est de mesure nulle. Supposons que $\mu\left(H \backslash S_{E}\right)>0$. Puisque $\varphi$ vérifie la condition $C_{0}$, il existe un ensemble mesurable $B \subset H \backslash S_{E}$ de mesure non nulle tel que $1_{B} \in L_{0}^{\varphi}(\Omega)$ dont tous les éléments sont dominés par un point $x_{0} \in H \backslash S_{E}$. Puisque $x_{0} \in H$, par définition de $H$ (cf. (28)) $x_{0}$ est dominé par tout point d'un ensemble mesurable $C \subset S_{E}$ de mesure non nulle tel que $1_{C} \in L_{0}^{\varphi}(\Omega)$. Alors on a

$$
\forall x \in B, \forall y \in C: \quad \varphi_{x} \prec \varphi_{y} .
$$

On en déduit en vertu du théorème 3.1 que l'ensemble $\left\{T\left(1_{C}\right): T \in \mathscr{L}\left[L_{0}^{\varphi}(C)\right.\right.$ $\left.L_{0}^{\varphi}(B)\right]$ \} est dense dans $L_{0}^{\varphi}(B)$. Comme $C \subset S_{E}$, il est clair que $1_{C} \in E$.

Soit $p_{C}$ l'opérateur de projection de $L_{0}^{\varphi}(\Omega)$ sur $L_{0}^{\varphi}(C)$. Alors pour tout opérateur linéaire continu $T$ de $L_{0}^{\varphi}(C)$ dans $L_{0}^{\varphi}(B)$, To $p_{C}$ est un opérateur linéaire continu de $L_{0}^{\varphi}(\Omega)$ dans $L_{0}^{\varphi}(B)$. Comme $1_{B} \in L_{0}^{\varphi}(B)$, cet élément est approchable par les $T \circ p_{C}\left(1_{C}\right)$ pour $T$ parcourant $\mathscr{L}\left[L_{0}^{\varphi}(C), L_{0}^{\varphi}(B)\right]$. D'où $1_{B} \in E$, car $E$ est fermé et invariant par tous les endomorphismes continus de $L_{0}^{\varphi}(\Omega)$. Il en résulte que $B \backslash S_{E}=B$ est négligeable, ce qui est une contradiction.

Donc $\mu\left(H \backslash S_{E}\right)=0$ et par conséquent

$$
E=L_{0}^{\varphi}\left(S_{E}\right)=L_{0}^{\varphi}\left(S_{E} \cap H\right)=L_{0}^{\varphi}(H) .
$$

Il est évident que dans ce cas $0<\mu(H)<\mu(\Omega)$.

THÉORẺME 3.5. Soient $\Omega$ un espace métrique séparable complet muni d'une mesure $\mu$ bornée régulière sans atome et $\varphi$ une fonction de Musielak-Orlicz sur $\Omega$ vérifiant les conditions $C_{0}$ et $C_{1}$, de plus telle que $\varphi(t, x)$ est une fonction de $t$ concave pour tout $x$ dans $\Omega$. Alors un sous-espace fermé non trivial $E$ de $L_{0}^{\varphi}(\Omega)$ est invariant pour tous les endomorphismes continus de $L_{0}^{\varphi}(\Omega)$ si et seulement si il existe un sous-ensemble mesurable héréditaire $H$ de mesure strictement comprise entre 0 et $\mu(\Omega)$ tel que $E=I_{0}^{\varphi}(H)$.

Démonstration. La nécessité de la condition est une conséquence de la proposition précédente. 
Quant à la suffisance, prenons un ensemble mesurable héréditaire quelconque $H \subset \Omega$ tel que $0<\mu(H)<\mu(\Omega)$. On démontre facilement par contraposition que

$$
\limsup _{t \rightarrow \infty} \frac{\varphi(t, y)}{\varphi(t, x)}=+\infty \quad \forall(x, y) \in H \times(\Omega \backslash H) .
$$

Mais $L_{0}^{\varphi}(\Omega)=L^{\varphi}(\Omega)$ (cf. Remarque 3) est une somme topologique directe de sous-espaces $L_{0}^{\varphi}(H)$ et $L_{0}^{\varphi}(\Omega \backslash H)$. Par conséquent, pour tout $u$ dans $L_{0}^{\varphi}(H)$ et pour tout $T$ dans l'algèbre $\mathscr{L}\left[L_{0}^{\varphi}(\Omega)\right]$ on a

$$
T u=v+w \quad \text { où } \quad v \in L_{0}^{\varphi}(H) \text { et } w \in L_{0}^{\varphi}(\Omega \backslash H) .
$$

La restriction de $T$ à $L_{0}^{\varphi}(H)$, soit $\left.T\right|_{L_{(}^{\varphi}(H)}$, et la projection $p_{\Omega \backslash H}$ de $L_{0}^{\varphi}(\Omega)$ sur $L_{0}(\Omega \backslash H)$ définie par

$$
p_{\Omega \backslash H}(u)=u \cdot 1_{\Omega \backslash H} \quad \forall u \in L_{0}^{\varphi}(\Omega)
$$

étant linéaires et continues, $\left.p_{\Omega \mid H} \circ T\right|_{L_{O}^{\varphi}(H)}$ est un opérateur linéaire continu de $L_{0}^{\varphi}(H)$ dans $L_{0}^{\varphi}(\Omega \backslash H)$. De plus, pour tout $u$ dans $L_{0}^{\varphi}(H)$, en tenant compte de (30), le théorème 2.3 implique que $\left(\left.p_{\Omega \mid H} \circ T\right|_{L_{\zeta}^{\varphi}(H)}\right)(u)=w=0$. Il en résulte que pour tout $u$ dans $L_{0}^{\varphi}(H)$ on a

$$
T u=\left.T\right|_{L_{(H)}^{\varphi}(H)}(u) \in L_{0}^{\varphi}(H) \quad \forall T \in \mathscr{L}\left[L_{0}^{\varphi}(\Omega)\right],
$$

ce qui prouve que $L_{0}^{\varphi}(H)$ est invariant par rapport à tous les endomorphismes continus de $L_{0}^{\varphi}(\Omega)$.

Remarque 6. Si $\varphi$ est une fonction de Musielak-Orlicz sur un espace mesuré $\Omega$ de mesure $\sigma$-finie sans atome telle que $\varphi(t, x)$ est une fonction de $t$ concave, les sous-espaces $L_{0}^{\varphi}(H)$ où $H$ est un sous-ensemble mesurable de $\Omega$ héréditaire tel que $0<\mu(H)$ et $\mu(\Omega \backslash H)>0$ sont invariants pour tous les endomorphismes continus de l'espace $L_{0}^{\varphi}(\Omega)$.

EXEMPLE 3. Soit

$$
\varphi^{0}(t, x)=t^{x} \text { pour } 0<x \leqslant 1 \text { et } t \geqslant 0 .
$$

Nous savons que $\varphi_{x_{1}}^{0} \prec \varphi_{x_{2}}^{0}$ si et seulement si $x_{1} \leqslant x_{2} . \varphi^{0}$ est une fonction de Musielak-Orlicz sur ]0, 1] telle que $\varphi^{0}(t, x)$ est une fonction de $t$ concave pour tout $x$ dans $] 0,1]$ et vérifiant les conditions $C_{0}$ et $C_{1}$ (cf. Exemple 1). Les seuls sous-espaces fermés non triviaux invariants pour tous les endomorphismes continus de $\left.\left.L_{0}^{\varphi^{0}}(] 0,1\right]\right)$ sont de la forme $\left.\left.L_{0}^{\phi}(] 0, x\right]\right)$ pour tout $x$ tel que $0<x<1$.

EXEMPLE 4. Si $p(x)$ est une fonction monotone définie sur $\boldsymbol{R}$ et à valeurs dans $] 0,1]$, posons

$$
\varphi^{1}(t, x)=t^{p(x)}
$$

$\varphi^{1}$ est aussi une fonction de Musielak-Orlicz telle que $\varphi^{1}(t, x)$ est une fonction de $t$ concave pour tout $x$ dans $\boldsymbol{R} . \varphi_{x}^{1} \prec \varphi_{y}^{1}$ si et seulement si $p(x) \leqslant p(y)$. Les sous-espaces $\left.\left.L_{0}^{\varphi^{1}}(]^{\prime}-\infty, x\right]\right)$ et $L_{0}^{\varphi^{1}}([x,+\infty D$ pour tout $x$ dans $\boldsymbol{R}$ sont invariants par rapport à tous les endomorphismes continus de $L_{0}^{\varphi_{0}^{1}}(\boldsymbol{R})$ lorsque $p$ est respectivement monotone croissante ou monotone décroissante.

Remerciements. Nous tenons à exprimer notre vive reconnaissance à $\mathrm{Ph}$ Turpin de l'Université de Paris-Sud (Orsay) pour l'aide qu'il nous a apportée pendant l'élaboration de cette note. Nos vifs remerciement s'adressent également à J. Trzeciak, rédacteur aux Éditions Scientifiques Polonaises, pour avoir amelioré la rédaction de la note lors de la correction des épreuves.

\section{Références}

[1] N. Bourbaki, Topologie Générale, vol. I, Hermann, Paris 1971

[2] L. Drewnowski and W. Orlicz, A note on modular spaces. XI, Bull. Acad. Polon. Sci. Sér. Sci. Math. Astronom. Phys. 16 (11) (1968), 877-882.

[3] P. R. Halmos, Measure Theory, Van Nostrand, Princeton 1950.

[4] N. J. Kalton, Transitivity and quotients of Orlicz spaces, Comment. Math., Tomus specialis in honorem Ladislai Orlicz, vol. I, 1978.

[5] N. J. Kalton and J. W. Roberts, A rigid subspace of $L_{0}$, Trans. Amer. Math. Soc. 266 (1981), 645-654

[6] G. Köthe, Topological Vector Spaces I, Springer, Berlin-Heidelberg-New York 1969

[7] C. M. Marle, Mesures et Probabilités, Hermann, Paris 1974.

[8] J. Musielak and W. Orlicz, Some remarks on modular spaces, Bull. Acad. Polon. Sci Sér. Sci. Math. Astronom. Phys. 7 (11) (1959), 661-668.

[9] -, -, On modular spaces, Studia Math. 18 (1959), 49-65.

[10] W. Orlicz, On spaces of $\varphi$-integrable functions, in: Proc. Intern. Symp. on Linear Spaces, Hebrew Univ. of Jerusalem, July 5-12, 1960, 357-365.

[11] H. L. Royden, Real Analysis, Macmillan, 1963.

[12] H. H. Schaefer, Banach Lattices and Positive Operators, Springer, New York-Heidelberg-Berlin 1974.

[13] P. Turpin. Opérateurs linéaires entre espaces d'Orlicz non localement convexes, Studia Math. 46 (1973), 153-165.

[14] -, Convexités dans les espaces vectoriels topologiques généraux, Dissertationes Math (Rozprawy Mat.) 131 (1976), 224 pp.

[15] -, Conditions de bornitude et espaces de fonctions mesurables, Studia Math. 56 (1976), 69-

[16] L. Waelbroeck, Topological Vector Spaces and Algebras, Lectures Notes in Math. 230, Springer, Berlin-Heidelberg-New York 1971

[17] -, A rigid topological vector space, Studia Math $\uparrow 59$ (1977), 227-234.

THE UNIVERSITY OF ZAMBIA

LUSAKA CAMPUS, DEPARTMENT OF MATHEMATICS

P.O. Box 32379, Lusaka, Zambia

Received May 2, 1985 